\title{
Two Glucose-Lowering Mechanisms of Canagliflozin Depending on Body Weight Changes in Drug-Naïve Subjects with Type 2 Diabetes
}

\author{
Eiji Kutoh $^{1,2,3} \cdot$ Asuka Wada $^{2} \cdot$ Teruma Murayama $^{1} \cdot$ Jyunka Hayashi $^{2}$
}

Published online: 15 October 2018

(c) The Author(s) 2018

\begin{abstract}
Objectives The aim of this study was to investigate the relations between the changes in body weight and those of glycemic and non-glycemic parameters in drug-naïve subjects with type 2 diabetes mellitus (T2DM) treated with canagliflozin monotherapy.

Methods Subjects received 50-100 mg/day canagliflozin monotherapy for 3 months $(n=36)$, and were then divided into two groups: (1) those who lost weight [changes in $(\Delta) \mathrm{BMI} \leq-0.45, p<0.00001$ : Group L(ost), $n=20)$; and (2) those who did not lose weight [ $\Delta \mathrm{BMI}>-0.45, p=$ non-significant: Group N(eutral), $n=16]$. At 3 months, the levels of glycemic and non-glycemic parameters were compared with those at baseline.

Results Significant reductions of BMI levels $(-2.1 \%, p<0.00001)$ were observed for the overall subjects. At baseline, fasting blood glucose (FBG) and HbA1c levels were significantly higher, and homeostasis model assessment-B (HOMA-B) levels were significantly lower in Group N versus Group L. Similar reductions of HbA1c (Group L: $9.54 \pm 2.58 \%$ to $7.54 \pm 1.27 \%$, $p<0.05$; Group N: $11.23 \pm 2.27 \%$ to $9.19 \pm 1.64 \%, p<0.0002$ ) and homeostasis model assessment-R (HOMA-R; Group L: $-32.3 \%, p<0.005$; Group N: $-36.5 \%, p<0.02)$ levels were seen in these two groups. However, other parameters showed distinct regulatory patterns. (1) Group L: significant reductions in uric acid (UA) levels $(-6.9 \%, p<0.02)$ were observed. Significant correlations between the changes in FBG and HOMA-R $(R=0.458, p<0.05)$ were seen. (2) Group N: significant increases in HOMA-B $(+69.4 \%, p<0.007)$ and reductions in free fatty acid (FFA; $-25.8 \%, p<0.02)$ levels were observed. Significant negative or positive correlations between the changes in $(\Delta)$ FBG and $\Delta$ HOMA-B $(R=-0.557, p<0.03)$, and between $\Delta$ FBG and $\triangle$ HOMA-R $(R=0.458, p<0.05)$ were seen.

Conclusions These results indicate that (1) body weight changes with canagliflozin were not associated with its glycemic efficacy; and (2) distinct glucose-lowering pathways may exist with canagliflozin, reducing insulin resistance in those who lose weight and enhancing $\beta$-cell function, as well as reducing insulin resistance, possibly via the decreased FFA levels, in those who do not lose weight.
\end{abstract}

Eiji Kutoh

ekuto@excite.com; ekuto-biomed@umin.ac.jp

1 Department of Clinical Research, Biomedical Center, Tokyo, Japan

2 Division of Diabetes and Endocrinology, Department of Internal Medicine, Gyoda General Hospital, Saitama, Japan

3 Division of Diabetes and Metabolism, Department of Internal Medicine, Higashitotsuka Memorial Hospital, Yokohama, Japan 


\section{Key Points}

Certain populations did not lose weight with canagliflozin treatment; however, these subjects had similar glucose-lowering levels in comparison with those who lost weight with this drug.

In those subjects who lost weight, canagliflozin was associated with improvements in the levels of metabolic parameters related to cardiovascular risk factors, including uric acid and triglyceride levels.

Canagliflozin may possess distinct, dual glucose-lowering mechanisms depending on body weight changes as follows. In subjects who lose weight, the degrees of insulin resistance decrease. By contrast, in subjects who do not lose weight, this drug reduces lipotoxicity via the decreased free fatty acid levels, thereby activating $\beta$-cell function and/or reducing insulin resistance.

\section{Introduction}

Sodium-glucose co-transporter 2 (SGLT-2) inhibitors are new oral hypoglycemic drugs for type 2 diabetes mellitus (T2DM) that increase glucose excretion into the urine [1-3]. Consistent with this mechanism of action, one of the most beneficial non-glycemic properties of SGLT-2 inhibitors is their effect on body weight [4]; however, it is frequently experienced by physicians and patients that body weight reductions of SGLT-2 inhibitors are not always linked to their glycemic efficacies as previously proposed [5].

Canagliflozin is one of the SGLT-2 inhibitors used in many countries $[6,7]$. Since chemical structures of SGLT-2 inhibitors are rather similar, canagliflozin was shown to possess similar pharmacological properties as other SGLT-2 inhibitors [8]. With the simple mechanism of excreting glucose into the urine, SGLT-2 inhibitors, including canagliflozin, were shown to ameliorate glucotoxicity [9], thereby ameliorating $\beta$-cell function and enhancing insulin sensitivity $[6,7]$. Furthermore, it was preliminarily reported that ipragliflozin, another SGLT-2 inhibitor, could downregulate free fatty acid (FFA) levels in certain populations, thereby affecting $\beta$-cell function and/or insulin sensitivity [5].

This study was a post hoc analysis of our previous work [10], and, in particular, explored the link between the changes in body weight and those of metabolic parameters with canagliflozin. This drug was used as monotherapy to reduce the influence of other drugs. Therefore, canagliflozin monotherapy was administered to drug-naïve subjects with T2DM. Body mass index (BMI) and other metabolic parameters were compared with baseline values at 3 months. No other drugs were administered during the study. Effects on glycemic and non-glycemic parameters were investigated in two groups, with or without body weight reductions.

\section{Subjects and Methods}

\subsection{Subjects}

The inclusion and exclusion criteria of subjects have been described previously [10]. Subjects were recruited from the outpatient department of the Department of Internal Medicine, Gyoda General Hospital, Saitama, Japan, and other associated hospitals of the first author (EK).

Female subjects $(n=10)$ only took $50 \mathrm{mg} /$ day of study drug owing to frequent female-specific adverse events (e.g. urogenital infections), while male subjects $(n=26)$ took $100 \mathrm{mg} /$ day. No other drugs were administered during the study. Subjects were encouraged to follow the exercise and diet as described [10]. Informed consents were obtained from patients and the protocol of this study was approved by the Ethical Committee/Institutional Review Board of Gyoda General Hospital. This study was conducted in accordance with the principles of Good Clinical Practice. In the case of unacceptable or undesirable therapeutic outcome, patients were free to leave therapy whenever they wished.

\subsection{Measurements}

The primary endpoint was changes in BMI levels from baseline to 3 months, while secondary endpoints included changes in fasting blood glucose (FBG), HbA1c, insulin, homeostasis model assessment-R (HOMA-R), homeostasis model assessment-B (HOMA-B), total cholesterol (T-C), triglyceride (TG), high-density lipoprotein cholesterol (HDLC), non-HDL-C, low-density lipoprotein cholesterol (LDLC), uric acid (UA), and FFA from baseline to 3 months. HbA1c values were assessed by the National Glycoprotein Standardization Program (NGSP) [11]. Blood was collected in the fasting state in the morning hours, and the standard technique was used to measure these parameters as described previously [10]. Subjects were divided into two groups: Group L ('lost', $n=20$ ) comprised patients who lost a significant amount of weight (change in BMI $[\Delta \mathrm{BMI}] \leq-0.45$; $p<0.00001$ ), and Group N ('neutral', $n=16$ ) comprised patients who did not lose weight $(\Delta \mathrm{BMI}>-0.45 ; p=$ nonsignificant). This cut-off value was the borderline where the changes in BMI levels become significant or non-significant.

\subsection{Data Analyses}

This study was a post hoc analysis of our previous work [10]. Since FFA was not measured in three male subjects from the 
original study [10], these subjects were excluded from the data analysis. Most of the statistical analysis was performed as described previously [10]. Descriptive statistics for all parameters studied included the mean changes from baseline to 3 months. The PAST program from the University of Oslo (https://folk.uio.no/ohammer/past/) was employed for statistical analysis. Multiple regression analysis was undertaken to identify any contributing factors relating to the changes in BMI levels with canagliflozin. The following independent variables were used: baseline levels of age, FBG, TG, nonHDL-C, UA, HOMA-R, HOMA-B, FFA and BMI. Throughout the statistical analysis, $p$ values $<0.05$ were considered statistically significant, whereas $p$ values of $0.05 \leq p<0.1$ were considered statistically insignificant but determined to show a tendency to potential differences or correlations.

\section{Results}

\subsection{Effects on Glycemic and Non-glycemic Parameters with Canagliflozin (All Subjects)}

For the overall subjects, significant reductions in FBG, HbA1c, BMI and HOMA-R levels were observed, while significant increases in HOMA-B levels were seen at 3 months (for each value and statistical significance, see Table 1). UA levels had a tendency to decrease. Little, if any, changes in lipid parameters, including T-C, TG, HDL-C, non-HDL-C, LDL-C and FFA levels, were noted. Multiple regression analysis was undertaken to identify contributing factors for the changes in $(\Delta)$ BMI (dependent variable) with canagliflozin, with the following independent variables being used: age, baseline level of FBG, BMI, TG, non-HDL-C, FFA, UA, HOMA-R and HOMAB. Among these factors, the baseline level of UA might be the responsible factor for the changes in BMI during canagliflozin treatment (Table 2). Indeed, nearly significant negative correlations were seen between the baseline UA levels and $\triangle \mathrm{BMI}$ with the post hoc simple regression analysis $(p=0.062)$ (Fig. 1).

Table 2 Factors associated with the changes $(\Delta)$ in BMI levels with canagliflozin

\begin{tabular}{lclcll}
\hline & Coeff. & Std. err. & $t$ & $p$ & $R^{2}$ \\
\hline Constant & 1.042 & 1.6562 & 0.62915 & 0.53474 & \\
Age & -0.004 & 0.01075 & -0.3726 & 0.71247 & 0.00241 \\
FBG & -0.0008 & 0.00311 & -0.2692 & 0.78991 & 0.03786 \\
Non-HDL & 0.00154 & 0.00329 & 0.4697 & 0.64248 & 0.01267 \\
TG & 0.00211 & 0.00154 & 1.3695 & 0.18256 & 0.07154 \\
BMI & -0.0159 & 0.03799 & -0.4193 & 0.67844 & 0.02254 \\
FFA & -0.238 & 0.41815 & -0.5691 & 0.5742 & 0.00041 \\
UA & -0.2554 & 0.12245 & -2.0859 & 0.04695 & 0.09837 \\
HOMA-R & 0.06011 & 0.06507 & 0.92382 & 0.36407 & 0.02227 \\
HOMA-B & -0.0044 & 0.00698 & -0.6373 & 0.52949 & 0.00985 \\
\hline
\end{tabular}

Multiple regression analysis was undertaken between $\triangle \mathrm{BMI}$ (dependent variable) and the baseline levels of the indicated parameters (independent variables)

$B M I$ body mass index
Table 1 Baseline characteristics and changes in glycemic and non-glycemic parameters with canagliflozin monotherapy in drug-naïve subjects with T2DM (overall subjects)

\begin{tabular}{llcll}
\hline & Baseline & 3 months & $p$ values & \% changes \\
\hline Age (years) & $51.4 \pm 14.0$ & & & \\
F/M & $10 / 26$ & & & \\
BMI & $27.59 \pm 5.56$ & $27.00 \pm 5.50$ & $<0.00001$ & -2.1 \\
FBG (mg/gl) & $207.6 \pm 66.7$ & $149.5 \pm 32.4$ & $<0.00001$ & -27.9 \\
HbA1c (\%) & $10.29 \pm 2.52$ & $8.27 \pm 1.66$ & $<0.00001$ & -19.6 \\
Insulin (mU/ml) & $9.60 \pm 7.17$ & $8.89 \pm 6.26$ & n.s. & -7.3 \\
HOMA-R & $4.82 \pm 9.15$ & $3.17 \pm 2.17$ & $<0.0002$ & -34.2 \\
HOMA-B & $31.16 \pm 30.12$ & $44.18 \pm 38.20$ & $<0.007$ & 41.7 \\
UA (mg/dl) & $5.50 \pm 1.21$ & $5.25 \pm 1.19$ & n.s. & -4.5 \\
T-C (mg/dl) & $214.8 \pm 38.7$ & $211.6 \pm 39.1$ & n.s. & -1.4 \\
TG (mg/dl) & $183.3 \pm 134.8$ & $168.7 \pm 95.8$ & n.s. & -7.9 \\
HDL-C (mg/dl) & $50.5 \pm 13.3$ & $52.3 \pm 12.2$ & n.s. & 3.5 \\
Non-HDL-C (mg/dl) & $164.2 \pm 38.3$ & $159.2 \pm 36.9$ & n.s. & -3 \\
LDL-C (mg/dl) & $139.4 \pm 37.8$ & $136.0 \pm 34.6$ & n.s. & -2.4 \\
FFA (eE/l) & $0.816 \pm 0.330$ & $0.714 \pm 0.384$ & n.s. & -12.5 \\
\hline
\end{tabular}

A paired Student's $t$ test was performed to analyze the changes in these parameters

$F$ female, $M$ male, $B M I$ body mass index, $F B G$ fasting blood glucose, HOMA-R homeostasis model assessment-R, HOMA-B homeostasis model assessment-B, $U A$ uric acid, $T-C$ total cholesterol, $T G$ triglyceride, $H D L-C$ high-density lipoprotein cholesterol, $L D L-C$ low-density lipoprotein cholesterol, $F F A$ free fatty acid, n.s. non-significant 
Fig. 1 Correlations between the changes in $\triangle \mathrm{BMI}$ and baseline UA levels. Simple regression analysis was performed between the indicated parameters (all subjects). $B M I$ body mass index, $U A$ uric acid

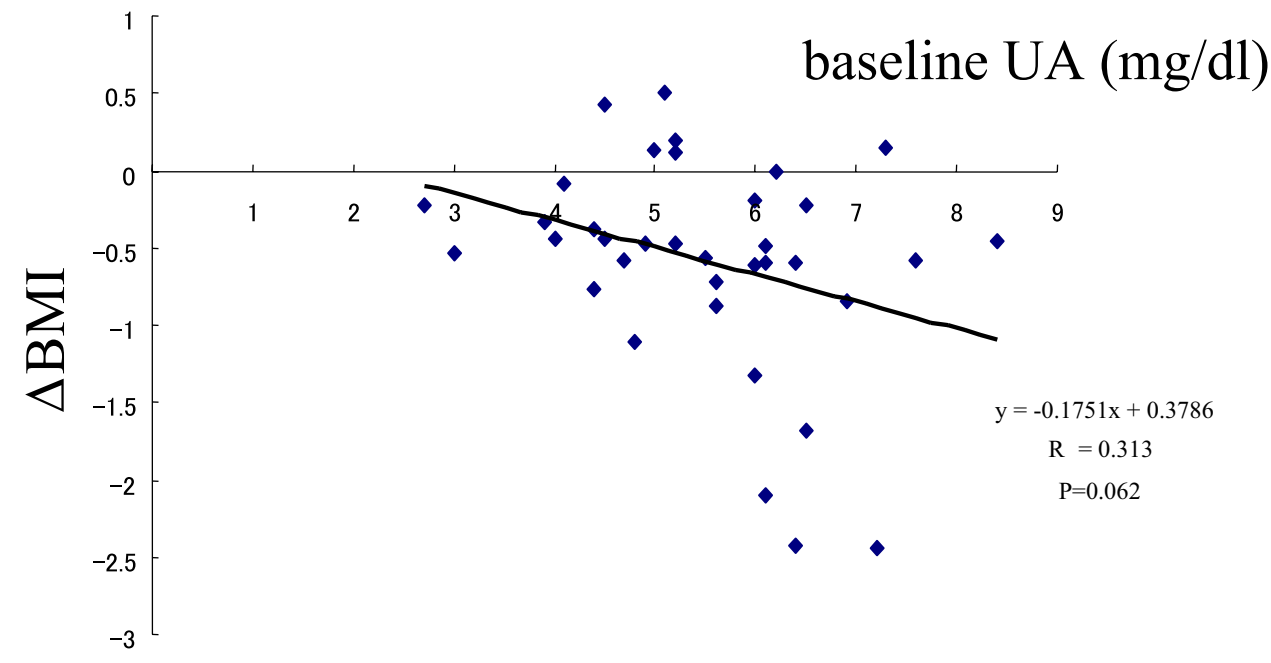

\subsection{Differential Regulations of Glycemic and Non-glycemic Parameters with Canagliflozin Depending on Body Weight Changes}

Subjects were then divided into two groups-those who significantly lost weight $(\Delta \mathrm{BMI} \leq-0.45, p<0.00001$; termed 'Group L', $n=20$ ) and those who did not lose weight $(\Delta \mathrm{BMI}>-0.45, p=$ non-significant; termed 'Group N', $n=16)$. This cut-off value of -0.45 for $\Delta \mathrm{BMI}$ is described in Sect. 2.2.

As indicated in Table 3, FBG and HbA1c levels were significantly higher in Group N versus Group L, while HOMA$B$ levels were significantly higher in Group L versus Group $\mathrm{N}$ at baseline. Other parameters showed no statistically significant differences at baseline. Similar reductions in FBG, HbA1c and HOMA-R levels were observed in these two groups (Table 4a, b) (with ANCOVA, no intergroup differences were noted in the changes in these parameters between these two groups, results not shown). However other parameters showed distinct regulatory patters as follows: Group L: significant reductions in BMI and UA levels were seen, and TG levels had a tendency to decrease (Table 4a); Group $\mathrm{N}$ : significant reductions in FFA and increases in HOMA-B levels were observed (Table 4b).

The next question was whether the reductions of glucose levels (assessed with FBG) were linked to the changes in insulin resistance (assessed with HOMA-R) and/or $\beta$-cell function (assessed with HOMA-B) in Groups L and N. To answer this question, simple regression analysis was performed as described in Sect. 2. Significant correlations were observed between $\triangle$ HOMA-R and $\triangle$ FBG in Group $\mathrm{L}$ (Table 5), while significant positive or negative correlations were seen between $\triangle \mathrm{HOMA}-\mathrm{R}$ and $\triangle \mathrm{FBG}$, or between $\Delta$ HOMA-B and $\Delta$ FBG, respectively, in Group N (Table 5).
Table 3 Baseline characteristics of glycemic and non-glycemic parameters between Groups L and N

\begin{tabular}{llll}
\hline & Group N & Group L & $p$ values \\
\hline Age (years) & $51.6 \pm 17.2$ & $51.2 \pm 13.8$ & n.s. \\
F/M & $3 / 13$ & $7 / 13$ & \\
BMI & $27.33 \pm 6.94$ & $27.80 \pm 4.19$ & n.s. \\
FBG (mg/dl) & $239.5 \pm 55.2$ & $182.1 \pm 65.1$ & $<0.01$ \\
HbA1c (\%) & $11.23 \pm 2,27$ & $9.54 \pm 2.58$ & $<0.05$ \\
insulin (mU/ml) & $8.68 \pm 7.62$ & $10.34 \pm 6.71$ & n.s. \\
HOMA-R & $5.22 \pm 4.67$ & $4.50 \pm 2.81$ & n.s. \\
HOMA-B & $18.88 \pm 16.84$ & $40.99 \pm 35.03$ & $<0.03$ \\
UA (mg/dl) & $5.18 \pm 1.41$ & $5.75 \pm 1.05$ & n.s. \\
T-C (mg/dl) & $214.3 \pm 42.1$ & $215.3 \pm 35.0$ & n.s. \\
TG (mg/dl) & $215.3 \pm 146.1$ & $157.7 \pm 73.6$ & n.s. \\
HDL-C (mg/dl) & $51.1 \pm 15.2$ & $50.1 \pm 11.1$ & n.s. \\
Non-HDL-C (mg/dl) & $163.1 \pm 38.4$ & $165.2 \pm 36.7$ & n.s. \\
LDL-C (mg/dl) & $133.4 \pm 43.2$ & $144.2 \pm 33.7$ & n.s. \\
FFA (eE/l) & $0.883 \pm 0.420$ & $0.762 \pm 0.249$ & n.s. \\
\hline
\end{tabular}

An unpaired Student's $t$ test was performed to compare the baseline values of the indicated parameters between these two groups

$F$ female, $M$ male, $B M I$ body mass index, $F B G$ fasting blood glucose, $H O M A-R$ homeostasis model assessment-R, HOMA-B homeostasis model assessment-B, $U A$ uric acid, $T-C$ total cholesterol, $T G$ triglyceride, $H D L-C$ high-density lipoprotein cholesterol, $L D L-C$ low-density lipoprotein cholesterol, FFA free fatty acid, n.s. non-significant

\section{Discussion}

\subsection{Body Weight Changes and Glycemic and Non-glycemic Efficacies with Canagliflozin}

Multiple regression analysis implicated that the baseline level of UA could be responsible for the changes in BMI levels with this drug (Table 2). This was further confirmed 
Table 4 Changes in glycemic and non-glycemic parameters with canagliflozin in (a) Group L, and (b) Group N

\begin{tabular}{|c|c|c|c|c|}
\hline & Baseline & 3 months & $p$ values & $\%$ changes \\
\hline \multicolumn{5}{|l|}{ (a) } \\
\hline Age (years) & $51.2 \pm 13.8$ & & & \\
\hline $\mathrm{F} / \mathrm{M}$ & $7 / 13$ & & & \\
\hline BMI & $27.80 \pm 4.19$ & $26.81 \pm 4.06$ & $<0.00001$ & -3.5 \\
\hline FBG (mg/dl) & $182.1 \pm 65.1$ & $135.7 \pm 26.6$ & $<0.005$ & -25.4 \\
\hline HbA1c (\%) & $9.54 \pm 2.58$ & $7.54 \pm 1.27$ & $<0.05$ & -20.9 \\
\hline Insulin (mU/ml) & $10.34 \pm 6.71$ & $9.40 \pm 6.88$ & n.s. & -9 \\
\hline HOMA-R & $4.50 \pm 2.81$ & $3.05 \pm 2.08$ & $<0.005$ & -32.2 \\
\hline HOMA-B & $40.99 \pm 35.03$ & $53.93 \pm 45.83$ & n.s. & 31.5 \\
\hline $\mathrm{UA}(\mathrm{mg} / \mathrm{dl})$ & $5.75 \pm 1.05$ & $5.35 \pm 1.17$ & $<0.05$ & -6.9 \\
\hline $\mathrm{T}-\mathrm{C}(\mathrm{mg} / \mathrm{dl})$ & $215.3 \pm 35.0$ & $212.2 \pm 37.6$ & n.s. & -1.4 \\
\hline $\mathrm{TG}(\mathrm{mg} / \mathrm{dl})$ & $157.7 \pm 73.6$ & $135.3 \pm 57.9$ & 0.09 & -14.2 \\
\hline HDL-C (mg/dl) & $50.1 \pm 11.1$ & $51.5 \pm 8.8$ & n.s. & 2.7 \\
\hline Non-HDL-C (mg/dl) & $165.2 \pm 36.7$ & $161.2 \pm 37.5$ & n.s. & -2.4 \\
\hline LDL-C (mg/dl) & $144.2 \pm 33.7$ & $141.7 \pm 34.6$ & n.s. & -1.7 \\
\hline FFA (eE/l) & $0.762 \pm 0.249$ & $0.762 \pm 0.418$ & n.s. & 0 \\
\hline \multicolumn{5}{|l|}{ (b) } \\
\hline Age (years) & $51.6 \pm 17.2$ & & & \\
\hline $\mathrm{F} / \mathrm{M}$ & $3 / 13$ & & & \\
\hline BMI & $27.33 \pm 6.94$ & $27.25 \pm 6.93$ & n.s. & -0.2 \\
\hline FBG (mg/dl) & $239.5 \pm 55.2$ & $166.6 \pm 33.4$ & $<0.0002$ & -30.4 \\
\hline HbA1c (\%) & $11.23 \pm 2.27$ & $9.19 \pm 1.64$ & $<0.0002$ & -18.1 \\
\hline Insulin (mU/ml) & $8.68 \pm 7.62$ & $8.25 \pm 6.07$ & n.s. & -4.9 \\
\hline HOMA-R & $5.22 \pm 4.67$ & $3.31 \pm 2.53$ & $<0.02$ & -36.5 \\
\hline HOMA-B & $18.88 \pm 16.84$ & $31.99 \pm 24.75$ & $<0.007$ & 69.4 \\
\hline $\mathrm{UA}(\mathrm{mg} / \mathrm{dl})$ & $5.18 \pm 1.41$ & $5.13 \pm 1.27$ & n.s. & -0.9 \\
\hline $\mathrm{T}-\mathrm{C}(\mathrm{mg} / \mathrm{dl})$ & $214.3 \pm 42.1$ & $210.2 \pm 41.9$ & n.s. & -1.9 \\
\hline TG (mg/dl) & $215.3 \pm 146.1$ & $210.5 \pm 127.9$ & n.s. & -2.2 \\
\hline HDL-C (mg/dl) & $51.1 \pm 15.2$ & $53.3 \pm 12.6$ & n.s. & 4.3 \\
\hline Non-HDL-C (mg/dl) & $163.1 \pm 38.4$ & $156.8 \pm 37.7$ & n.s. & -3.8 \\
\hline LDL-C (mg/dl) & $133.4 \pm 43.2$ & $128.9 \pm 36.9$ & n.s. & -3.3 \\
\hline FFA (eE/l) & $0.883 \pm 0.420$ & $0.655 \pm 0.297$ & $<0.02$ & -25.8 \\
\hline
\end{tabular}

A paired Student's $t$ test was performed to analyze the changes in the parameters in these two groups $F$ female, $M$ male, $B M I$ body mass index, $F B G$ fasting blood glucose, HOMA- $R$ homeostasis model assessment-R, HOMA- $B$ homeostasis model assessment-B, $U A$ uric acid, $T-C$ total cholesterol, $T G$ triglyceride, $H D L-C$ high-density lipoprotein cholesterol, $L D L-C$ low-density lipoprotein cholesterol, $F F A$ free fatty acid, n.s. non-significant
Table 5 Correlations between the changes in glycemic parameters and those of insulin resistance (HOMA-R) and $\beta$-cell function (HOMA-B)

\begin{tabular}{lcc}
\hline & $R$ & $p$ values \\
\hline Group L & 0.458 & $<0.05$ \\
$\Delta$ HOMA-R vs $\Delta$ FBG & & \\
Group N & -0.557 & $<0.03$ \\
$\Delta$ HOMA-B vs $\Delta$ FBG & 0.607 & $<0.02$ \\
$\Delta$ HOMA-R vs $\Delta$ FBG & \\
\hline
\end{tabular}

by the post hoc simple regression analysis showing that nearly significant correlations were noted between these parameters (Fig. 1). When the subjects were divided into two groups, depending on the changes in BMI (Groups L and $\mathrm{N}$ ), only those in Group L had a small but still significant reduction in UA levels, although the baseline UA levels were within the normal range (but insignificantly higher than those of Group N) (Table 3). These backgrounds implicate that UA may be linked to weight reduction during canagliflozin treatment. The mechanism of body weight reduction with SGLT-2 inhibitors is to facilitate fat metabolism via the increased glucose excretion into the urine. While UA is produced mainly in the liver, recent results have shown that UA is also produced in the fat tissue and is increased 
in obesity [12]. This could be a potential mechanism of the reduced UA levels in those who lose weight with SGLT-2 inhibitors, including canagliflozin.

Interestingly, similar glycemic efficacies were seen in subjects with (Group L) or without (Group N) body weight reductions (Table 4a, b). This result implicates that the glycemic efficacy of canagliflozin is not linked to the reductions of body weight as shown with another SGLT-2 inhibitor [5]. However, those who lose weight with canagliflozin may have more advantages than those who do not with respect to some of the non-glycemic parameters, including UA and TG (Table 4a). Elevated UA and/or TG levels are potential risk factors for cardiovascular or cerebrovascular diseases [13]. It has recently been shown that canagliflozin had beneficial outcomes on some cardiovascular aspects (CANVAS) [14]. An understanding of the mechanisms of the favorable effects on the heart would be of importance. Subanalysis of the subjects in terms of weight changes in CANVAS could be particularly interesting.

\subsection{Differential Modulation of Insulin Resistance and $\beta$-Cell Function in the Glycemic Efficacy of Canagliflozin Depending on Body Weight Changes}

It is well-known that losing weight is effective for better glycemic control; however, as presented in this study, similar glycemic efficacies were seen with canagliflozin irrespective of the changes in body weight (Table $4 a, b$ ). What are the underlying mechanisms of this phenomenon? Blood glucose levels are determined by the modulation of insulin resistance and $\beta$-cell function [15]. In those who lost weight, the degrees of insulin resistance (assessed by HOMA-R) were significantly reduced, and good correlations were seen between the changes in HOMA-R and FBG (Group L) (Tables 4a, 5). On the other hand, in those who did not lose weight, levels of $\beta$-cell functions (assessed by HOMA-B) were significantly elevated and good negative correlations were observed between the changes in HOMA-B and FBG (Group N) (Tables 4b, 5). Furthermore, in this group, the degrees of insulin resistance were significantly reduced, and good correlations were seen between the changes in HOMA-R and FBG (Tables 4b, 5).

One interesting question is the potential mechanism of increased $\beta$-cell function/decreased insulin resistance in those without weight loss (Group N). Significant reductions of FFA levels were observed in Group N (Table 4b). Elevated FFA levels are known to increase insulin resistance $[16,17]$ and to decrease $\beta$-cell function through lipotoxicity $[18,19]$. Modulation of insulin resistance and $\beta$-cell function via the decreased FFA levels could be one of the potential mechanisms of good glycemic efficacies of canagliflozin, although no changes in body weight were noted in Group N.
It is generally believed that FFA levels are increased with SGLT-2 inhibitors because they shift substrate utilization from carbohydrate to lipids [20]. Therefore, one could easily imagine that those who lose weight (Group L) have elevated FFA levels via the decreased TG levels [20]. Given this background, FFA levels are not increased in those who have no changes in weight (Group N). The precise mechanism of how canagliflozin can downregulate FFA levels in those without weight changes remains to be investigated. Collectively, two glucose-lowering mechanisms could be present with canagliflozin depending on body weight changes: (1) reducing insulin resistance by weight loss (Group L); and (2) enhancing $\beta$-cell function/reducing insulin resistance possibly by downregulating FFA levels (Group N) (as illustrated in Fig. 2). It remains to be investigated whether or not similar results would be obtained with other SGLT-2 inhibitors.

\subsection{Limitations and Strengths of the Study}

Some limitations exist with this study. This was an observational (but prospective) study with rather small numbers of study participants and a short duration. Lack of a control group, for example using subjects treated with diet/exercise alone, is also a drawback of this study. However, considering the design of the study (monotherapy with drug-naïve subjects), it is likely that the observed changes in glycemic and non-glycemic parameters were mainly caused by canagliflozin. Another limitation is the lack of multiplicity used to account for the numerous associations and differences tested. Further well-designed studies, such as randomized, double-blind, placebo-controlled, longer duration studies

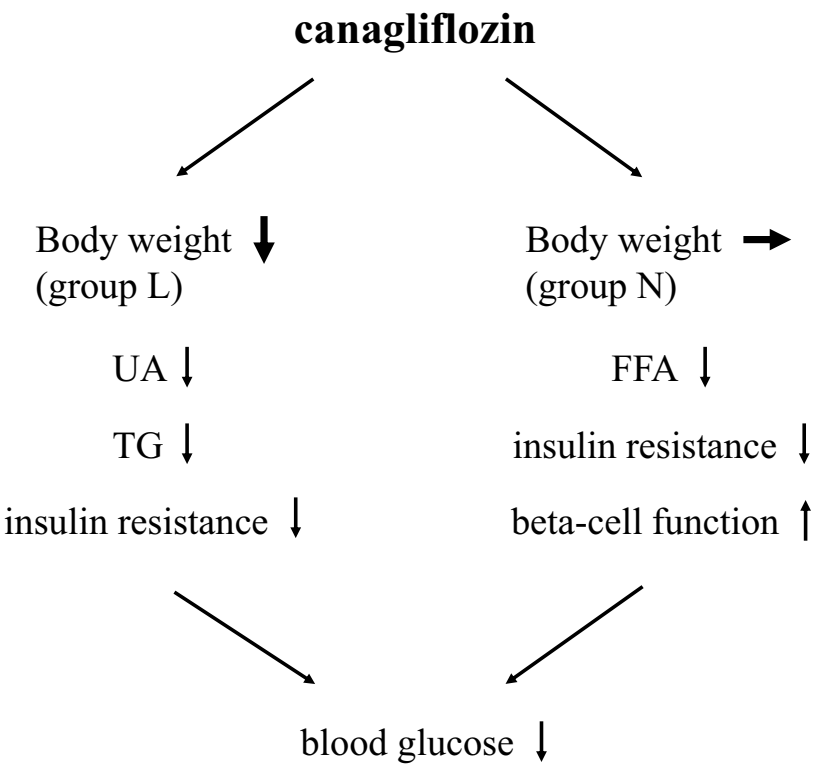

Fig. 2 Schematic representation of glycemic efficacy of canagliflozin depending on the changes in body weight 
with increased numbers of subjects, will be necessary to augment the findings of this study.

\section{Conclusions}

The contents of this article add the following new knowledge: canagliflozin may possess distinct, dual glucose-lowering mechanisms depending on body weight changes. In subjects who lose weight, the degrees of insulin resistance decrease. By contrast, in subjects who do not lose weight, canagliflozin reduces FFA levels and may thereby activate $\beta$-cell function and reduce insulin resistance. Similar glycemic efficacies were seen in these two populations. However, in those who lose weight, canagliflozin is associated with improvements in the levels of metabolic parameters, including UA and TG levels, in comparison with those who do not lose weight.

Acknowledgements The authors thank Drs. Kenji Kawashima, Takashi Suzuki, Rika Kusudo, Takashi Nakamura and Alexandra Kuto for discussions and useful comments.

\section{Compliance with ethical standards}

Informed consent All subjects provided informed consent.

Conflict of interest Eiji Kutoh, Asuka Wada, Teruma Murayama, and Jyunka Hayashi declare that no conflict of interests exist regarding this manuscript.

Ethical approval The studies were conducted in accordance with the Declaration of Helsinki and International Conference on Harmonisation Good Clinical Practice (ICH GCP) guidelines.

Author contributions EK participated in the design of the study and acquisition of the data, performed the statistical analysis and drafted the manuscript. AW, TM and JH made substantial contributions to the conception and design of the study and helped draft the manuscript. All authors read and approved the final version of the manuscript.

Funding No funding was received for this study.

Open Access This article is distributed under the terms of the Creative Commons Attribution-NonCommercial 4.0 International License (http://creativecommons.org/licenses/by-nc/4.0/), which permits any noncommercial use, distribution, and reproduction in any medium, provided you give appropriate credit to the original author(s) and the source, provide a link to the Creative Commons license, and indicate if changes were made.

\section{References}

1. Abdul-Ghani MA, Norton L, Defronzo RA. Role of sodium-glucose cotransporter 2 (SGLT 2) inhibitors in the treatment of type 2 diabetes. Endocr Rev. 2011;32(4):515-31.
2. Whalen K, Miller S, Onge ES. The role of sodium-glucose cotransporter 2 inhibitors in the treatment of type 2 diabetes. Clin Ther. 2015;37(6):1150-66.

3. DeFronzo RA, Davidson JA, Del Prato S. The role of the kidneys in glucose homeostasis: a new path towards normalizing glycaemia. Diabetes Obes Metab. 2012;14(1):5-14.

4. Scheen AJ, Paquot N. Metabolic effects of SGLT-2 inhibitors beyond increased glucosuria: a review of the clinical evidence. Diabetes Metab. 2014;40(6 Suppl 1):S4-11.

5. Kutoh E, Murayama T, Wada A, Hirate M. Distinct glucoselowering mechanisms of ipragliflozin depending on body weight changes. Drugs R D. 2016;16(4):369-76.

6. Rosenthal N, Meininger G, Ways K, Polidori D, Desai M, Qiu R, et al. Canagliflozin: a sodium glucose co-transporter 2 inhibitor for the treatment of type 2 diabetes mellitus. Ann N Y Acad Sci. 2015;1358:28-43.

7. Brunton S, Reid TS. Canagliflozin, a sodium glucose co-transporter 2 inhibitor, for the management of type 2 diabetes. Hosp Pract (1995). 2014;42(3):96-108.

8. Kuriyama C, Ueta K, Arakawa K. Pharmacological and clinical profile of a novel SGLT2 inhibitor canagliflozin hydrate $\left(\right.$ CANAGLU $\left.^{\circledR}\right)$. Nihon Yakurigaku Zasshi. 2015;146(6):332-41.

9. Takahara M, Shiraiwa T, Matsuoka TA, Katakami N, Shimomura I. Ameliorated pancreatic $\beta$ cell dysfunction in type 2 diabetic patients treated with a sodium-glucose cotransporter 2 inhibitor ipragliflozin. Endocr J. 2015;62(1):77-86.

10. Kutoh E, Wada A, Murayama T, Takizawa Y. Canagliflozin as an initial therapy in drug-naïve subjects with type 2 diabetes mellitus: a potential involvement of atherogenic lipids in its glycemic efficacy. Drugs R D. 2017;17(2):313-20.

11. Miedema K. Towards worldwide standardisation of HbA1c determination. Diabetologia. 2004;47(7):1143-8.

12. Tsushima Y, Nishizawa H, Tochino Y, Nakatsuji H, Sekimoto R, Nagao $\mathrm{H}$, et al. Uric acid secretion from adipose tissue and its increase in obesity. J Biol Chem. 2013;288(38):27138-49.

13. Fukuda H, Haruyama Y, Nakade M, Muto T. Relationship between lifestyle and change of cardiovascular risk factors based on a five-year follow-up of employees in Japan. Ind Health. 2007;45(1):56-61.

14. Neal B, Perkovic V, Mahaffey KW, de Zeeuw D, Fulcher G, Erondu N, CANVAS Program Collaborative Group, et al. Canagliflozin and cardiovascular and renal events in type 2 diabetes. N Engl J Med. 2017;377(7):644-57.

15. Kutoh E, Fukushima T. Insulin-dependent actions of pioglitazone in newly diagnosed, drug-naïve patients with type 2 diabetes. Endocrine. 2009;35(3):333-40.

16. Boden G. Fatty acids and insulin resistance. Diabetes Care. 1996;19(4):394-5.

17. Olson AL. Insulin resistance: cross-talk between adipose tissue and skeletal muscle, through free fatty acids, liver $\mathrm{X}$ receptor, and peroxisome proliferator-activated receptor- $\alpha$ signaling. Horm Mol Biol Clin Investig. 2013;15(3):115-21.

18. Giacca A, Xiao C, Oprescu AI, Carpentier AC, Lewis GF. Lipidinduced pancreatic $\beta$-cell dysfunction: focus on in vivo studies. Am J Physiol Endocrinol Metab. 2011;300(2):E255-62.

19. Unger RH. Lipotoxicity in the pathogenesis of obesity-dependent NIDDM. Genetic and clinical implications. Diabetes. 1995;44(8):863-70.

20. Yokono M, Takasu T, Hayashizaki Y, Mitsuoka K, Kihara R, Muramatsu Y, et al. SGLT2 selective inhibitor ipragliflozin reduces body fat mass by increasing fatty acid oxidation in highfat diet-induced obese rats. Eur J Pharmacol. 2014;727:66-74. 